\title{
Sexually transmitted infections among girls aged 1 to 15 years, presumed victims of sexual abuse in Thies/ Senegal: about 98 cases
}

\section{Pauline Dioussé ${ }^{1,3}$, Mariétou Thiam", ${ }^{1,2}$ Agbogbenkou Tevi Deladem Lawson', Haby Dione ${ }^{1}$, Lamine Gueye², Mariama Bammo³, Thierno Abdoul Aziz Diallo', Fatou Seck1, Ndiaga Gueye', Amadou Mactar Gueye1, Ramatoulaye Diagne Gueye1, Mamadou Lamine Cissé1,2, Mame Thierno Dieng ${ }^{4}$, Bernard Marcel Diop ${ }^{1}$, Mamadou Mourtalla Ka ${ }^{1}$}

${ }^{1}$ Faculty of Health Sciences, University of Thies, Senegal, ${ }^{2}$ Department of Obstetrics and Gynecology, Thies regional Hospital, Senegal, ${ }^{3}$ Department of Dermatology, Cheikh Anta Diop University, Dakar, Senegal, ${ }^{4}$ Department of Dermatology, Thies Regional Hospital, Senegal.

Corresponding author: Dr. Pauline Dioussé, E-mail: paudiousse@yahoo.fr

\begin{abstract}
Background: Sexually transmitted infections among girls remain a health problem, which, outside the perinatal period, occur most often in the context of sexual abuse. The objectives of this study were to determine the epidemiologic, clinical, para clinical and therapeutic aspects. Patients and Methods: This was a retrospective study carried out from the 1st January 2011 to 31 st December 2018 (8 years). All files of girls under the age of 15 presumed to be victims of sexual abuse were included. The data collected comprised socio demographic, clinical, para clinical, and therapeutic variables. Results: 143 patients were consulted for sexual abuse, among which 98, that is, $73 \%$ were children. The average age was 10.4 years \pm 3.35 years ( $2-15$ years). The port of entry was vaginal penetration in 40 cases $(40.8 \%)$. The time to consultation was 29 days \pm 127 days. The retro viral and HBs antigen serology performed in $97 \%$ of the cases was negative. Syphilis serology was positive only in one case. The germs isolated were: Mycoplasma (2\%); Ureaplasma, Chlamydia, Trichomonas vaginalis, Gardnerella vaginalis, and Candida albicans were found at $1 \%$ each. Two (2\%) of the children had received prior anti retro viral prophylaxis. Conclusion: In children, the diagnostic of sexual abuse remains difficult. And the resulting sexually transmitted infections are even more so. Confirmation requires specific methods, especially since there are legal proceedings.
\end{abstract}

Key words: Sexually transmitted infections; Child sexual abuse; Senegal

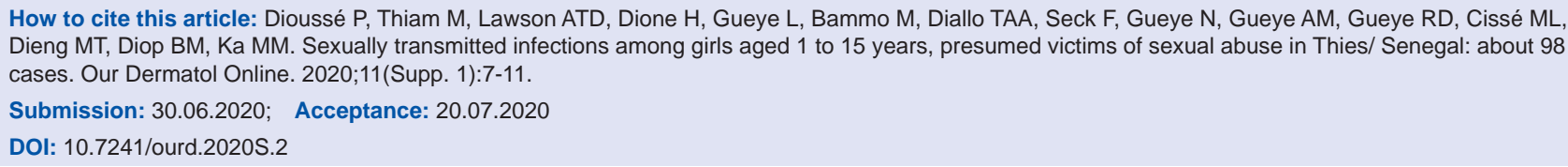




\title{
Les infections sexuellement transmissibles chez les filles âgées de 1 à 15 ans victimes présumées d'abus sexuels à Thiès/Sénégal : à propos de 98 cas
}

\section{Pauline Dioussé1,3, Mariétou Thiam,1,2, Agbogbenkou Tevi Deladem Lawson', Haby Dione', Lamine Gueye ${ }^{2}$, Mariama Bammo ${ }^{3}$, Thierno Abdoul Aziz Diallo', Fatou Seck', Ndiaga Gueye', Amadou Mactar Gueye', Ramatoulaye Diagne Gueye', Mamadou Lamine Cissé1,2, Mame Thierno Dieng ${ }^{4}$, Bernard Marcel Diop ${ }^{1}$, Mamadou Mourtalla Ka ${ }^{1}$}

${ }^{1}$ Faculty of Health Sciences, University of Thies, Senegal, ${ }^{2}$ Department of Obstetrics and Gynecology, Thies regional Hospital, Senegal, ${ }^{3}$ Department of Dermatology, Cheikh Anta Diop University, Dakar, Senegal, ${ }^{4}$ Department of Dermatology, Thies Regional Hospital, Senegal.

Corresponding author: Dr. Pauline Dioussé, E-mail: paudiousse@yahoo.fr

\begin{abstract}
RÉSUMÉ
Introduction: Les infections sexuellement transmissibles (IST) chez les filles demeurent un problème de santé, qui en dehors de la période péri natale, surviennent le plus souvent dans un contexte d'abus sexuels. Les objectifs étaient d'en déterminer les aspects épidémiologiques, cliniques, para cliniques, thérapeutiques. Méthodes et Patients: Il s'agissait d'une étude rétrospective menée du ler Janvier 2011 au 31 Décembre 2018 (8 ans). Etaient inclus tous les dossiers de filles dont l'âge est inférieur ou égal à 15 ans victimes présumées d'abus sexuel. Les données recueillies comprenaient les variables sociodémographiques, cliniques, para cliniques, thérapeutiques. Résultats: 134 patientes avaient consulté pour abus sexuel dont 98 enfants soit 73\%. La moyenne d'âge était de 10,4 ans $\pm 3,35$ ans (2 ans-15 ans). La voie de pénétration était vaginale dans 40 cas $(40,8 \%)$. Le délai moyen de consultation était de 29 jours \pm 127 jours. La sérologie rétrovirale et l'antigène HBs faites dans $97 \%$ des cas étaient négatifs. La sérologie syphilitique était positive dans un cas. Les germes retrouvés étaient: Mycoplasma (2\%) ; les autres germes : Ureaplasma, Chlamydiae, Trichomonas vaginalis, Gardnerella vaginalis, Candida albicans étaient retrouvés chacun dans 1\%. Deux (2\%) enfants avaient reçu une prophylaxie antirétrovirale. Conclusion: Chez l'enfant, la certitude d'un abus sexuel reste difficile, les IST qui en découlent le sont encore plus. La confirmation nécessite des méthodes spécifiques d'autant plus qu'il existe des poursuites judiciaires.
\end{abstract}

Mots clés: Infections sexuellement transmissibles; Abus sexuel d'enfants; Sénégal

\section{INTRODUCTION}

Les infections sexuellement transmissibles (IST) chez les filles demeurent un problème de santé. En dehors de la période péri natale, elles surviennent le plus souvent dans un contexte d'abus sexuels. Ces derniers sont un véritable fléau touchant toutes les sociétés du monde entier. L'Organisation Mondiale de la Santé (OMS) a estimé en 2004 que la prévalence mondiale des violences sexuelles sur enfant était plus élevée chez les filles soit environ $27 \%$ [1].
Cette association décrite en Europe, en Amérique, en Arabie $[2,3,4,5,6]$ a fait l'objet de nombreuses études menées en Afrique Australe [7,8]. Ces dernières années, elle était documentée en Afrique Centrale et de l'Ouest $[9,10,11]$. Au Sénégal, la plupart des études faites l'ont été dans la capitale et s'appesantissaient plus sur les abus sexuels [12]. La survenue d'IST au décours de ces actes n'était pas suffisamment décrite ce qui justifie notre travail.

Les objectifs étaient de déterminer les aspects épidémiologiques, cliniques, paracliniques et

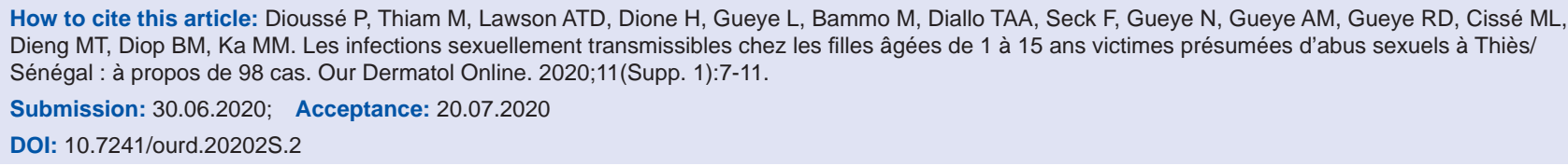


thérapeutiques des IST chez les filles reçues pour présumés abus sexuels.

\section{MATERIALS AND METHODS}

Type et cadre d'étude: Il s'agissait d'une étude rétrospective menée dans le service de Gynécologie Obstétrique de l'Hôpital Régional de Thiès

Période d'étude: Elle était menée du ler Janvier 2011 au 31 Décembre 2018, soit une période de 8 ans.

Critères d'inclusion: étaient inclus tous les dossiers de filles dont l'âge est inférieur ou égal à 15 ans victimes présumées d'abus sexuel, reçues en urgence à la consultation externe.

Critères de non inclusion: les dossiers inexploitables n'étaient pas inclus.

\section{Variables Étudiées}

Les paramètres suivants étaient étudiés :

- Sociodémographiques : la fréquence, l'âge, le niveau scolaire, l'adresse, la voie de pénétration, la notion de port de préservatif et d'éjaculation.

- Cliniques : le délai moyen de consultation, les traumatismes génitaux (cutanéomuqueuses)

- Les résultats des examens complémentaires : la recherche de l'antigène $\mathrm{HBs}$, la sérologie syphilitique, la sérologie rétrovirale et le prélèvement vaginal.

- Le traitement administré.

\section{Analyse Statistique}

Pour des raisons éthiques, les numéros des dossiers, le nom et l'adresse des victimes n'ont pas été divulgués

Lanalyse statistique des données était faite à partir du logiciel EPI Info version 3.5.4. (CDC Atlanta). Pour comparer les proportions, le test du Chi Carré était utilisé avec un seuil de significativité $\mathrm{p}<0,05$.

\section{Ethics Statement}

This study was performed on records of patients.

\section{RESULTS}

Durant la période d'étude 134 patientes avaient consulté en urgence pour abus sexuel dont 98 enfants, les enfants représentaient ainsi $73 \%$ des victimes présumés d'abus sexuel. La moyenne d'âge était de 10,4 ans $\pm 3,35$ ans [2 ans-15 ans]. Cinquante et une fille ( $52 \%$ ) étaient au niveau primaire et $17(17,3 \%)$ au niveau secondaire. Elles habitaient dans la ville de Thiès dans 37 cas $(37,8 \%)$, en zone périurbaine dans 46 cas (47\%) dans un autre département dans 15 cas $(15,3 \%)$. La voie de pénétration était vaginale dans 40 cas $(40,8 \%)$, anale dans 2 cas (2\%), le reste non précisé. L'agresseur ne portait pas de préservatif dans 92 cas $(93,9 \%)$ et il avait éjaculé dans 20 cas $(20,4 \%)$.

Le délai moyen de consultation était de 29 jours \pm 127 jours. Les traumatismes des muqueuses génitales étaient retrouvés dans 42 cas (43\%).

Sur le plan des examens complémentaires, la sérologie rétrovirale faite dans $97 \%$ des cas était négative, l'antigène $\mathrm{HBs}$ faite dans les mêmes proportions était négatif. La sérologie syphilitique faite dans 41 cas (42\%) était positive dans un cas chez une fille de 7 ans. Le prélèvement vaginal faite dans 95 cas (97\%) avait retrouvé des germes dans 15 cas (15,3\%). Les IST retrouvées étaient classées en fonction de l'âge de la victime (Tableau I).

Le traitement était à base d'antibiotiques dans 19 cas $(19,3 \%)$. Deux $(2 \%)$ enfants avaient reçu une prophylaxie antirétrovirale.

\begin{tabular}{|c|c|c|c|c|}
\hline $\begin{array}{l}\text { Infections } \\
\text { retrouvées }\end{array}$ & Germes & $\begin{array}{c}\text { Effectifs } \\
\mathrm{N}\end{array}$ & Pourcentage & $\begin{array}{l}\text { Age victime } \\
\text { (an) }\end{array}$ \\
\hline \multirow[t]{8}{*}{ Bactériennes } & Staphylocoques & 2 & 2,0 & 3-11 \\
\hline & $\begin{array}{l}\text { Germes non } \\
\text { spécifique }\end{array}$ & 3 & 3,0 & $5-11-15$ \\
\hline & Ureaplasma & 1 & 1,0 & 15 \\
\hline & $\begin{array}{l}\text { Ureaplasma et } \\
\text { Mycoplasma }\end{array}$ & 1 & 1,0 & 12 \\
\hline & Mycoplasma & 2 & 2,0 & $11-15$ \\
\hline & $\begin{array}{l}\text { Ureaplasma et } \\
\text { Chlamydiae }\end{array}$ & 1 & 1,0 & 7 \\
\hline & $\begin{array}{l}\text { Chlamydiae et } \\
\text { Mycoplasma }\end{array}$ & 1 & 1,0 & 15 \\
\hline & $\begin{array}{l}\text { Treponema } \\
\text { pallidum }\end{array}$ & 1 & 1,0 & 7 \\
\hline $\begin{array}{l}\text { Bactériennes } \\
\text { et } \\
\text { parasitaires }\end{array}$ & $\begin{array}{l}\text { Ureaplasma et } \\
\text { Gardnerella }\end{array}$ & 1 & 1,0 & 14 \\
\hline \multirow[t]{2}{*}{ Parasitaires } & $\begin{array}{l}\text { Trichomonas } \\
\text { vaginalis }\end{array}$ & 1 & 1,0 & 3 \\
\hline & $\begin{array}{l}\text { Gardnerella } \\
\text { vaginalis }\end{array}$ & 1 & 1,0 & 14 \\
\hline Mycosiques & $\begin{array}{l}\text { Candida } \\
\text { albicans }\end{array}$ & 1 & 1,0 & 13 \\
\hline
\end{tabular}




\section{DISCUSSION}

Le diagnostic médical d'abus sexuel chez les enfants reste difficile dans notre contexte. Les équipes doivent tenir compte des allégations de l'enfant, de certains comportements non spécifiques [13]. Elles doivent tenir compte aussi des signes physiques spécifiques d'abus sexuels. Le diagnostic est aisé si les allégations de la victime sont corrélées à l'existence de la notion de pénétration et de signes physiques. Cette notion de pénétration était de $43 \%$ dans notre série, $70 \%$ au Zimbabwe [7]. Joyce dans son étude, a noté une prévalence des blessures de 21,4\% (73 sur 340) chez des enfants examinés de manière aigus [14]. Dans la littérature, les auteurs ont trouvé que parmi les victimes prouvées d'abus sexuels, plus de la moitié avaient un examen physique normal [15]. Ce dernier pourrait s'expliquer en partie par le long délai de consultation comme dans notre série. Ce long délai pourrait entrainer un processus de réparation de l'hymen et faire disparaitre les signes physiques d'abus sexuels. D'autre part, certains abus n'impliquent pas de contact sexuel. A cela s'ajoute le fait que dans notre société, les abus sexuels sont toujours un sujet tabou pour la famille de la victime et sont surtout considérés comme une source de déshonneur social, personnel et familial. Et si l'agresseur a des liens de parentés avec la victime, l'abus sexuel est souvent étouffé par la famille.

Quant au diagnostic des IST, leur présence chez l'enfant nécessite des précautions dans l'interprétation. Les différents types d'IST n'ont pas la même valeur prédictive positive d'abus sexuel d'enfants. A cela s'ajoute le fait que le diagnostic biologique des IST en routine, n'est pas aisé dans notre région. Dans notre série, sur 98 filles, $10(10,2 \%)$ avaient une IST tandis que Girardet et al a noté que sur 485 filles, 8,2\% avaient une IST [4]. Il existe une notion de pénétration chez tous les enfants présentant une IST dans notre série, mais il n'y a pas eu de lien statistique significatif entre le traumatisme muqueux et la survenue d'IST.

Pitche et al, dans sa méta-analyse, concluait que la présence chez l'enfant de certains germes comme Chlamydiae, Trichomonas et le gonocoque était très probablement liée à un abus sexuel [16]. Linfection à C. trachomatis chez les enfants est souvent asymptomatique et peut persister pendant des mois à des années [5]. Elle était de $3.1 \%$ dans la série de Girardet et al [4]. Pour Chlamydia, le sérodiagnostic était la méthode utilisée dans notre série car étant la plus accessible. Cependant l'interprétation d'un examen sérologique reste difficile non seulement en raison de la réponse immune propre à chaque individu et de la sensibilité de la technique elle-même. Aussi, il est probable qu'il existe de faux positifs par réaction croisée du fait de l'existence du trachome dans notre région. Le trachome est une kérato-conjonctivite, contagieuse, d'évolution chronique évoluant jusqu'à la cécité dû à des infections répétées à Chlamydia trachomatis. Au Sénégal, la prévalence du trachome actif est de 10,8\% dans la population générale et de 14,2\% dans la région de Thiès [17]. Lidéal serait le diagnostic direct qui repose sur l'isolement de la bactérie par culture cellulaire. Même si la culture est considérée comme une référence pour la détection de C. trachomatis, la sensibilité peut varier considérablement d'un laboratoire à l'autre. Lisolement de C. trachomatis dans la culture de tissus doit être confirmé par l'identification microscopique des inclusions intra cytoplasmiques caractéristiques, de préférence avec un anticorps monoclonal conjugué à la fluorescéine spécifique de l'espèce [5].

Quant au Trichomonas vaginalis, il était de $5.9 \%$ dans la série de Girardet et al [4] et de $1 \%$ dans notre série. Il faut interpréter avec précaution la présence de trichomonas dans les échantillons d'urine des enfants. Parce que la morphologie des Penta trichomonas (Trichomonas) hominis, un flagellé intestinal non pathogène, est très similaire à celle de Trichomonas vaginalis [18].

Pour d'autres germes tels que ceux de la syphilis, l'abus sexuel est probable, il existe le risque de transmission mère-enfant. La syphilis était positive chez $0.3 \%$ des enfants dans l'étude de Girardet et al [4], dans notre série 1 fille âgée de 7 ans était positive. Le risque de confusion majeur dans le diagnostic de la syphilis chez les enfants au-delà de la période néonatale existe. Il est important de différencier l'infection acquise et celle congénitale. La syphilis acquise doit être suspectée chez les enfants symptomatiques ou séropositifs chez qui aucune preuve de syphilis congénitale n'a été documentée. Chez les femmes enceintes au Sénégal, la sérologie syphilitique est systématique durant les consultations prénatales. Seulement l'infection congénitale ne peut être exclue en période péri natale. Aussi, certaines manifestations cliniques de la syphilis congénitale peuvent se chevaucher avec celles de la syphilis acquise. Au Sénégal, du fait de l'accessibilité des antibiotiques dans les postes de santé, il n'existe plus des tréponématoses non vénériennes qui pourraient fausser la sérologie. Et en cas de positivité, le dosage quantitatif est recommandé pour vérifier si c'est une infection récente évolutive ou une cicatrice sérologique. D'où 
l'importance d'une bonne interprétation car des résultats faussement positifs peuvent avoir des conséquences juridiques et sociales graves. Il en de même pour la sérologie VIH. Elle était revenue positive 3,6\% dans la série de Faye Dieme et al [12]. Dans notre série, elle était de $0 \%$ après le premier prélèvement. Il n'y a pas eu de cas d'abus sexuels en rapport avec une purification de l'infection par le VIH par l'agresseur. Aucune patiente de notre série, n'avait bénéficié d'une réévaluation de son statut sérologique à 3 et 6 mois alors que c'est une recommandation du programme de lutte contre le VIH/ SIDA. Cet état de fait constitue une défaillance dans la prise en charge dans notre série et serait certainement dû au fait que les parents se focalisent plus sur l'aspect juridique au point où qu'ils ne reviennent plus une fois le certificat médical établi. Aussi, du fait du caractère rétrospectif de l'étude, le délit retenu par le tribunal et les sanctions n’ont pas été précisés.

La présence de Gardnerella est le signe d'un abus sexuel possible selon Pitche [16]. Nolan dans son étude a noté que 30\% d'entre eux étaient asymptomatiques [18]. Dans les cas de vulvovaginite causée par Candida sp, Les rapports sexuels ne sont pas la principale forme de transmission, puisque ces organismes peuvent faire partie de la flore endogène, leur apparition chez les enfants doit avoir une approche bien définie [19].

Les limites de l'étude sont le type rétrospectif, les difficultés de diagnostic biologique de certitude de certains germes d'IST en routine au Sénégal particulièrement à Thiès.

\section{CONCLUSION}

Chez l'enfant, la certitude d'un abus sexuel reste difficile. Et les IST qui en découlent le sont encore plus. La confirmation nécessite des méthodes spécifiques d'autant plus qu'il existe des poursuites judiciaires.

\section{Statement of Human and Animal Rights}

All procedures followed were in accordance with the ethical standards of the responsible committee on human experimentation (institutional and national) and with the Helsinki Declaration of 1975, as revised in 2008 .

\section{Statement of Informed Consent}

Informed consent was obtained from all patients for being included in the study.

\section{REFERENCES}

1. Thomas A, Forster G, Robinson A, Rogstad K. National guideline for the management of suspected sexually transmitted infections in children and young people. Arch Dis Child. 2003; 88:303-11.

2. Häuser W, Schmutzer G, Brähler E, Glaesmer H. Maltreatment in childhood and adolescence - results from a survey of a representative sample of the general German population. Deutsches Aerzteblatt Internat. 2011;108:287-94.

3. May-Chahal C, Herczog M. L'abus sexuel des enfants en Europe, Editions du Conseil de l'Europe, Strasbourg, 2003.

4. Girardet RG, Lahoti S, Howard LA, Fajman NN, Sawyer MK, Driebe EM, et al. Epidemiology of sexually transmitted infections in suspected child victims of sexual assault. Am Acad Ped. 2009;124:79-86.

5. Margaret R. Hammerschlag. sexual assault and abuse of children. Oxford J Med Health Clin Inf Dis. 2011;53:103-9.

6. Al Thukair AA, Sallout DH, Abdulghani DW, Al Afandi DT, Al Johi HA, Al Otaibi HO, et al. Spectrum of viral skin infections in patients attending the dermatology clinic at King Fahd Hospital of the University in Alkhobar, Kingdom of Saudi Arabia during the period 2010 - 2014. Our Dermatol Online. 2017;8:260-3.

7. Nhundu TJ, Shumba A. The nature and frequency of reported cases of teacher perpetrated child sexual abuse in rural primary schools in Zimbabwe. Child Abuse Negl. 2001; 25:1517-34.

8. Sumner SA, Mercy A, Saul J, Motsa-Nzuza N, Kwesigabo G, Buluma R, et al. Prevalence of sexual violence against children and use of social services-seven countries, 2007-2013. MMWR Morb Mortal Wkly Rep. 2015;64:565-9.

9. Markwei U, Mamle Tetteh P. Unpacking the ethics of access and safety of participants and researchers of child sexual abuse in Ghana. Child Geograph. DOI: 10.1080/14733285.2020.1786500.

10. Badoe E. A critical review of child abuse and its management in Africa. Afr J Emerg Med. 2017;7(Suppl):S32-5.

11. Oluwatoyin E, Akin-Odanye. Prevalence and management of child sexual abuse cases presented at Nigerian hospitals: A systematic review. J Health Soc Scien. 2018;3:109-24.

12. Faye Dieme ME, Traore AL, Gueye SMK, Moreira PM, Diouf A, Moreau JC. Profil épidémioclinique et prise en charge des victimes d'abus sexuels à la clinique gynécologique et obstétricale du CHU de Dakar. J Gyn Obst Biol Reprod. 2008;37:358-64.

13. Borg K, Snowdon C, Hodes D. A resilience-based approach to the recognition and response of child sexual abuse. Paediat Child Health. 2019;29:6-14.

14. Joyce A, Adams MD, Karen J, Farst MD, Nancy D, Kellogg MD. interpretation of medical findings in suspected child sexual abuse: an update for 2018. J Ped Adol Gynecol. 2018;31:225-31.

15. Adams JA. Medical evaluation of suspected child sexual abuse: 2011 Update. J Child Sex Abus. 2011;20:588-605.

16. Pitche P. Abus sexuels d'enfants et infections sexuellement transmissibles en Afrique Sub-Saharienne. Med Trop. 2005;65:570-74.

17. Sarr B, Sissoko M, Fall M, Alemayehu W, Bakhtiari A, Bovill S, et al. Prevalence of Trachoma in Senegal: Results of Baseline Surveys in 17 Districts. Ophthalmic Epidemiol. 2018; 25(sup1):41-52.

18. Nolan MS, Cruz AT, Erickson T. Retrospective chart analysis of child and adolescent trichomonas vaginalis infection in houston, texas. J Pediatric Infect Dis Soc. 2020;9:75-81.

19. Xu M, Liu H, Glick S, Khachemoune A. Perianal lesions in children: an updated review. Am J Clin Dermatol. 2017;18:343-54.

Copyright by Pauline Dioussé, et al. This is an open access article distributed under the terms of the Creative Commons Attribution License, which permits unrestricted use, distribution, and reproduction in any medium, provided the original author and source are credited. Source of Support: Nil, Conflict of Interest: None declared. 